\title{
Awakening tumor immunity
}

\section{By Lev Osherovich, Senior Writer}

Researchers at The University of Texas MD Anderson Cancer Center have determined why many cancer vaccines fail to elicit a robust $\mathrm{T}$ cell response-the cells hover near the vaccination site instead of migrating to the tumor. ${ }^{1}$ The surprisingly simple solution is switching the vaccine formulation to a different carrier.

Vaccination with peptide antigens typically elicits both antibody- and cell-based immune responses that are mediated by B cells and $\mathrm{T}$ cells, respectively. A robust response by $\mathrm{CD} 8^{+}$cytotoxic $\mathrm{T}$ cells is especially important in fighting tumors.

Ideally, a tumor-derived antigen vaccine would cause antigenpresenting cells (APCs) such as dendritic cells (DCs) to pick up the antigen

"The phenomenon of getting $T$ cell infiltration and sequestration at the vaccination site and absence of $T$ cells at the tumors is a very important finding. We now know the problem, and there's now a hypothesis to address this." -Benjamin Chen, Immune Targeting Systems Ltd. and carry it to lymph nodes, where they would then activate $B$ cells that make tumor-targeting antibodies and $\mathrm{CD}^{+}$cytotoxic $\mathrm{T}$ cells that hunt tumor cells.

In practice, raising antibodies against tumor antigens is easy, but getting a robust $\mathrm{T}$ cell response has proven challenging.

The only marketed cancer vaccine in the U.S.-Dendreon Corp.'s Provenge sipuleucel-T for prostate cancer-takes a shortcut to eliciting a strong $\mathrm{T}$ cell response.

Provenge therapy involves extracting and activating patient-derived DCs ex vivo, then reintroducing the DCs into patients in large enough numbers to promote tumor-targeting $\mathrm{T}$ cell activity.

Direct in vivo vaccination could help avoid the cost and complexity of autologous cell therapies like Provenge but would only be effective if a stronger T cell response could somehow be elicited.

Thus, Willem Overwijk, associate professor of melanoma medical oncology at MD Anderson, set out to understand why typical tumor vaccines lead to poor $\mathrm{T}$ cell responses.

He said prior preclinical and clinical studies have shown that cancer vaccines typically cause an initial burst of $\mathrm{T}$ cell activity, but the response quickly peters out.

\section{Failure to launch}

Overwijk's team vaccinated a mouse model for melanoma with a short frag- ment of the melanoma antigen silver homolog (SILV; PMEL17; GP100) in a solution of incomplete Freund's adjuvant (IFA), a commonly used mineral oil-based carrier.

As expected, the formulation elicited a strong B cell response but only a weak $\mathrm{T}$ cell response.

To understand why the vaccine-induced $\mathrm{T}$ cells were not very effective, Overwijk's team used a luciferase-based imaging system to look at the localization of $\mathrm{T}$ cells in tumor-bearing mice. Instead of migrating to the tumors, the antigen-specific $\mathrm{T}$ cells lingered around the vaccine injection site for weeks.

"We saw that T cells were getting hung up at the injection site," said Overwijk. "They expanded in number and hung out in the blood near the injection site. Very few of them went to the tumor sites."

Overwijk found that IFA did not readily disperse in the bloodstream and instead formed a long-lived depot of antigen that trapped T cells. Instead of homing to tumor cells, the T cells were sequestered near the vaccination site, formed a dense granuloma-like mass, became inactive and eventually underwent apoptosis.

"We think it's the long persistence of the vaccine that leads to this effect. The mineral oil is not biodegradable," he noted.

To overcome this problem, Overwijk's team experimented with various vaccine formulations that are shorter lived than IFA. The team settled on a formulation of GP100 and a cocktail of adjuvants suspended in a saline buffer.

With the new saline-based formulation, "the vaccine site becomes cleared of antigen in 4-5 days rather than 90 days with IFA," said Overwijk.

Mice vaccinated with the saline-based formulation showed $\mathrm{T}$ cell migration away from the vaccination site and toward the tumor (see Figure 1, "Improving tumor vaccine formulation"). The result was greater tumorspecific $\mathrm{T}$ cell responses at the tumor site than those seen when using IFAbased controls.

Findings were reported in Nature Medicine.

\section{Cancer and beyond}

Benjamin Chen, executive chairman of Immune Targeting Systems Ltd., said Overwijk's findings are potentially relevant to a variety of T cell-targeted vaccines for cancer and possibly other indications.

"It's been known that the IFA-like adjuvant used in humans can cause granulomas and necrosis at injection sites," but the significance of these structures was unclear, said Chen. "The phenomenon of getting T cell infiltration and sequestration at the vaccination site and absence of $\mathrm{T}$ cells at the tumors is a very important finding. We now know the problem, and there's now a hypothesis to address this."

Immune Targeting is developing self-adjuvanting, T cell-targeted peptide vaccines. A Phase IIa trial of the company's most advanced product-Flunisyn influenza A vaccine (FP-01.1) - met its primary endpoint of $\mathrm{T}$ cell induction. The company also has preclinical vaccine candidates for $\mathrm{HBV}$ and an undisclosed cancer indication.

Cornelis Melief, CSO of vaccine maker ISA Pharmaceuticals B.V., suspects that the carrier may not be the only culprit that hinders $\mathrm{T}$ cell migration. He noted that the poor efficacy of the mineral oil-based vaccine 

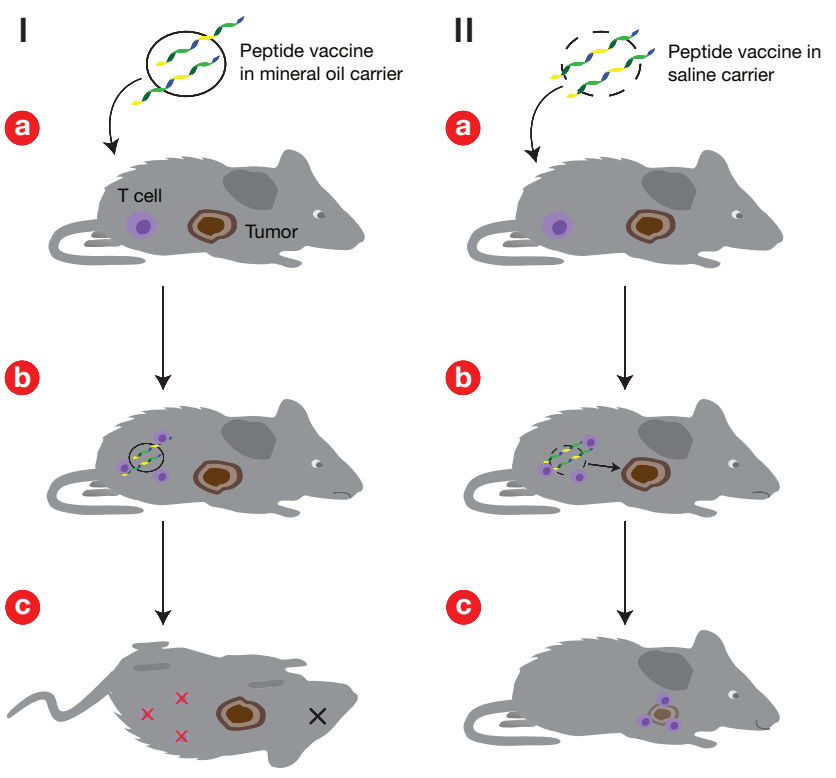

Figure 1. Improving tumor vaccine formulation. Hailemichael et al. have shown that a widely used adjuvant leads to $\mathrm{T}$ cell inactivity that impairs the efficacy of cancer vaccines and have devised a workaround.

A typical T cell-targeted cancer vaccine is formulated in a mineral oil-based carrier (I[a]). In a mouse tumor model, subcutaneous injection of this formulation draws $T$ cells to the injection site (I[b]), where they accumulate instead of migrating toward the tumor. These locally trapped $T$ cells eventually undergo apoptosis (I[c]), leading to tumor growth and death.

Hailemichael et al. formulated peptide antigens in a salinebased carrier (II[a]) and observed transient localization of T cells to the injection site followed by migration of the $T$ cells toward the tumor (II[b]), leading to an improved antitumor immune response (II[c]).

in the paper could result from the excessively short peptides used in the study.

ISA's vaccines use long peptides in an IFA-like carrier. The company's ISA-HPV-01, a therapeutic vaccine for HPV, is expected to start Phase II testing this year for advanced cervical cancer and is in Phase I/II trials for anal intraepithelial neoplasia.

Melief said the short GP100 antigen peptide used by Overwijk's team binds to MHC I receptors found on a multitude of cells, including the $\mathrm{T}$ cells themselves. As a result, vaccination with this short peptide can overstimulate the $\mathrm{T}$ cells and discourage proper presentation of antigens by DCs and other APCs.

Many researchers prefer using short-peptide antigens because they are easier to work with than longer peptides and do not require additional processing by APCs.

Melief believes a better approach is to use long-peptide antigens. Long peptides do not readily bind to MHC I and can only be taken up by DCs, which are less numerous than other cell types, including $\mathrm{T}$ cells. Instead of the excessive local response caused by short peptides, long peptides are carried from the injection site by DCs to lymph nodes, leading to a more measured and productive immune response.

"MHC I-binding peptides are lousy vaccines because they exogenously load onto all cells with class I MHC," said Melief. "This leads to a tolerizing mode of vaccination. If you are going to deliver short peptides, Overwijk clearly shows that the only way is to provide them along with strong adjuvants and saline."

Last year, Melief and collaborators at the Leiden University Medical Center reported that long-peptide vaccines induce an effective antitumor $\mathrm{T}$ cell response and tumor regression in a mouse model for HPV even when formulated with an IFA-like carrier. ${ }^{2}$

Likewise, Melief noted that the supplementary data in Overwijk's paper suggest that when the team used longer peptides, the melanoma vaccine appeared to work well even in IFA.

Overwijk said Melief is correct that "when given in IFA, long peptides are superior to short peptides, possibly because the intensity of antigen presentation of long peptides is lower."

However, he said, "we still see eventual T cell dysfunction after vaccination with long peptides in IFA, probably since the persistence of antigen is still too long."

Chen said the challenge now for vaccine makers is to maximize the exposure of antigens to APCs without causing long-term T cell sequestration. He said optimal local persistence of the antigen may vary from one vaccine to another.

"You want to attract antigen-presenting cells to come in and pick up the antigen and prime T cells, but you don't want the depot to be around for too long," said Chen. "You need to make sure the T cells get primed and home to where they are needed as quickly as possible."

Overwijk said a saline-based carrier is probably too short-lived to produce optimal DC responses. His team is now testing a variety of water-soluble carriers and materials including gels, microsomes and nanoparticles. The goal is to deliver the antigen in a way that mimics acute viral infection, which is familiar territory for the immune system.

"We're currently working with industry and academia to try a variety of particle-based, intermediate-release formulations," said Overwijk. "We haven't found the ideal formulation yet, but we think we want it to be around for around five days, which is about as long as a virus hangs around."

Overwijk did not disclose the companies with whom he is working. He did not patent his findings.

Osherovich, L. SciBX 6(12); doi:10.1038/scibx.2013.280

Published online March 28, 2013

\section{REFERENCES}

1. Hailemichael, Y. et al. Nat. Med.; published online March 3, 2013; doi:10.1038/nm.3105

Contact: Willem W. Overwijk, The University of Texas MD Anderson Cancer Center, Houston, Texas

e-mail: woverwijk@mdanderson.org

2. van Duikeren, S. et al. J. Immunol. 189, 3397-3403 (2012)

\section{COMPANIES AND INSTITUTIONS MENTIONED}

Dendreon Corp. (NASDAQ:DNDN), Seattle, Wash.

Immune Targeting Systems Ltd., London, U.K.

ISA Pharmaceuticals B.V., Leiden, the Netherlands

Leiden University Medical Center, Leiden, the Netherlands

The University of Texas MD Anderson Cancer Center,

Houston, Texas 